\title{
Hypotensive Epidural Anesthesia Reduces Blood Loss in Pelvic and Sacral Bone Tumor Resections
}

\author{
Alex K. Freeman BSc, MBChB, Chris J. Thorne MBChB, \\ C. Louie Gaston MD, Richard Shellard FRCA, Tom Neal FRCA, \\ Michael C. Parry MD, FRCS, Robert J. Grimer FRCS, \\ Lee Jeys MSc, FRCS
}

Received: 31 July 2015/Accepted: 19 April 2016/Published online: 12 May 2016

(C) The Association of Bone and Joint Surgeons \& 2016

\begin{abstract}
Background Resection of pelvic and sacral tumors can cause severe blood loss, complications, and even postoperative death. Hypotensive epidural anesthesia has been used to mitigate blood loss after elective arthroplasty, but to our knowledge, it has not been studied as an approach that might make resection of pelvic and sacral tumors safer. Questions/purposes The purposes of this study were (1) to compare the blood loss and blood product use for patients undergoing pelvic and sacral tumor surgery under standard anesthesia or hypotensive epidural anesthesia; (2)
\end{abstract}

Each author certifies that he, or a member of his immediate family, has no funding or commercial associations (eg, consultancies, stock ownership, equity interest, patent/licensing arrangements, etc) that might pose a conflict of interest in connection with the submitted article.

All ICMJE Conflict of Interest Forms for authors and Clinical Orthopaedics and Related Research $\AA$ editors and board members are on file with the publication and can be viewed on request.

Each author certifies that his or her institution approved or waived approval for the human protocol for this investigation and that all investigations were conducted in conformity with ethical principles of research.

This work was performed at The Royal Orthopaedic Hospital, Birmingham, UK.

A. K. Freeman, C. J. Thorne

School of Clinical and Experimental Medicine, University of

Birmingham, Edgbaston, UK

C. L. Gaston, R. Shellard, T. Neal, M. C. Parry ( $₫)$,

R. J. Grimer, L. Jeys

The Royal Orthopaedic Hospital NHS Foundation Trust, Bristol

Road South, Birmingham B31 2AP, UK

e-mail: michaelcparry@hotmail.com; michael.parry3@nhs.net

L. Jeys

School of Life and Health Sciences, Aston University,

Birmingham, UK to assess the frequency of end-organ damage with the two techniques; and (3) to compare 90-day mortality between the two techniques.

Methods Between 2000 and 2014, 285 major pelvic and sacral resections were performed at one center. A total of $174(61 \%)$ had complete data sets for analysis of blood loss, transfusion use, complications, and mortality at 90 days. Of those, 102 (59\%) underwent hypotensive epidural anesthesia, whereas the remainder received standard anesthetic care. The anesthetic approach was determined by the anesthetists in charge of the case with hypotensive epidural anesthesia exclusively performed by one of two subspecialty trained anesthetists as their routine for major pelvic or sacral surgery. The groups were comparable in terms of potential confounding variables such as age, gender, tumor volume, and operation performed. Hypotensive epidural anesthesia was defined as a technique using an extensive epidural block up to T2-3 dermatome, peripherally administered low-concentration intravenous adrenaline infusion, and using unimpeded spontaneous respiration to achieve controlled hypotension, precise rate control of the heart, and enhanced velocity of venous return, all aggregated thus to minimize blood loss during pelvic surgery while preserving vital perfusion. The groups were assessed for perioperative blood loss calculated from pre- and postsurgery hemoglobin and transfusion use as well as postoperative complications, morbidity, and mortality at 90 days.

Results There was less mean blood loss in the hypotensive epidural anesthesia group (1457 mL, SD 1721, 95\% confidence interval [CI], 1114-1801 versus $2421 \mathrm{~mL}, \mathrm{SD}$ 2297, 95\% CI, 1877-2965; $p=0.003$ ). Patients in the hypotensive epidural anesthesia group on average received fewer packed red cell transfusions (2.7 units, SD 2.9, 95\% CI, 2.1-3.2 versus 3.9 units, SD 4.4, 95\% CI, 2.9-5.0; 
$p=0.03)$. There were no differences in the proportions of patients experiencing end-organ injury $(7 \%, \mathrm{n}=$ seven of 102 versus $6 \%, n=$ four of $72 ; p=0.72$ ). With the numbers available, there was no difference in 90 -day mortality rate between groups $(1.9 \%, \mathrm{n}=$ two of 102 versus $1.3 \%, \mathrm{n}=$ one of $72 ; \mathrm{p}=0.77$ ).

Conclusions We found that hypotensive epidural anesthesia resulted in less blood loss, fewer transfusions, and no apparent increase in serious complications in pelvic and sacral tumor surgery performed in the setting of a highvolume tertiary sarcoma referral hospital. We recommend that further collaborative studies be undertaken to confirm our results with hypotensive epidural anesthesia in surgery for pelvic tumors.

Level of Evidence Level III, therapeutic study.

\section{Introduction}

Blood loss during pelvic tumor surgery can be severe [1], in one study varying between 2.5 and nearly $5 \mathrm{~L}$ [5]. Hemorrhagic hypovolemia resulting in allogeneic transfusion is associated with increased morbidity after nononcological surgery [8, 9, 12]. Allogeneic blood transfusions after oncological surgery may increase the risk of postoperative infection, and they may even increase the risk of cancer recurrence, perhaps an effect of immunosuppression and immune modulation [23]. In an attempt to minimize blood loss and the need for transfusion, many techniques for managing blood loss during pelvic surgery have emerged including preoperative erythropoietin, preoperative iron supplementation, hypotensive anesthesia, and intraoperative antifibrinolytic drugs.

In our institution, we have adopted a system of hypotensive epidural anesthesia. The technique, which involves a reduction in blood pressure while maintaining flow through stabilization of cardiac output, has demonstrated efficacy in lower limb orthopaedic surgery [7, 17$20,22]$, where it has been shown to reduce blood loss and transfusion reaction. Hypotensive epidural anesthesia provides sympathetic blockade by means of a high epidural anesthetic and sustained cardiac output by means of an adrenaline infusion. This results in reduced peripheral venous bleeding mediated by venous hypotension as a result of decreased peripheral resistance while maintaining perfusion of the vital organs and distal extremities. We have used this technique, together with standard anesthetic techniques, for a number of years for pelvic and sacral tumor resection. Tachycardia from intraoperative hypovolemia maybe masked with the technique and careful monitoring of blood loss during surgery is crucial. Despite these risks, studies have shown no increased incidence of end-organ damage after hypotensive epidural anesthesia, even in patients with existing comorbidities [7, 11, 17].

However, to our knowledge, no research has evaluated hypotensive epidural anesthesia to determine whether it can decrease blood loss or transfusion use during pelvic sarcoma surgery. Likewise, to our knowledge, no study has evaluated this approach to see whether it may increase the likelihood of end-organ damage, a possible complication of underperfusion or mortality after pelvic tumor surgery.

The primary aims of this study were to (1) compare the blood loss and blood product use between hypotensive epidural anesthesia and a standard anesthetic technique during pelvic and sacral tumor resection; (2) to assess the frequency of end-organ injury (acute kidney injury, myocardial ischemia, and cerebrovascular accidents); and (3) to compare the 90-day mortality after pelvic and sacral tumor resection between hypotensive epidural anesthesia and standard anesthetic techniques.

\section{Patients and Methods}

We undertook a retrospective, observational study of pelvic and sacral bone tumor cases at a single tertiary orthopaedic hospital (The Royal Orthopaedic Hospital, Birmingham, UK). The prospectively maintained institutional database was used to identify all patients who underwent a hemipelvectomy, sacrectomy, or hindquarter amputation between January 1, 2000, and August 31, 2014. A total of 285 patients were identified. The single exclusion criterion was the absence of data. A total of $111(39 \%)$ patients were excluded for this reason. Seventy-five patients had incomplete data, in which all the fields required were not completely documented in the anesthetic chart or hardcopy notes. For example, the estimated blood loss was unable to be calculated because the cases were before electronic record of blood results and their paper records of either preoperative or postoperative blood tests were not available. The patient hard-copy notes were inaccessible in a further 36 patients who had died and their hospital notes were not kept on-site at the time of our analysis. From a national registry, we know these patients did not die within 90 days of the index operation. The remaining total of 174 patients were included in this study. The study was registered and approved with the hospital institutional review board.

\section{Intervention}

Patients received one of two anesthetic techniques: hypotensive epidural anesthesia $(n=102$ [59\%]) or a 
standard anesthetic technique $(\mathrm{n}=72[41 \%]$; Table 1$)$. The anesthetic technique used was dependent on the anesthetist who was performing the case. The hypotensive epidural anesthesia group was exclusively performed by the two coauthors (RS, TN), whereas the standard anesthetic was used by other anesthetists in the hospital. The distribution of surgical operations between the two groups showed no statistical difference (Table 1) and was only determined by the anesthetist rostered to perform the anesthesia on the day of surgery.

The hypotensive epidural anesthesia technique uses a more extensive epidural block than usual, which acts as a cardiac sympathectomy, a peripherally administered lowconcentration intravenous adrenaline infusion for chronotropic effect, and unimpeded spontaneous respiration, which avoids cyclical positive pressure in the pelvic veins with the goal of achieving controlled hypotension, precise rate control of the heart, and enhanced velocity of venous return to the heart to minimize blood loss while preserving perfusion to vital end organs. Induction was performed with propofol with either ketamine or midazolam. Patients received a combined high spinal (between L2/3 and L4/5) with $0.5 \%$ bupivacaine and epidural (between T11/12 and L1/2) anesthesia with an initial bolus of $0.75 \%$ levobupivacaine administered through the epidural needle to achieve a cardiac sympathectomy block up to $\mathrm{T} 2 / 3$ dermatome. Patients remained spontaneously breathing on a laryngeal mask airway avoiding positive pressure ventilation. Anesthesia was maintained either intravenously (using propofol plus either remifentanil or ketamine) or using gaseous agents (sevoflurane/desflurane). A continuous infusion of adrenaline $(1: 250,000)$ was administered through a peripheral venous line to maintain cardiac output and a target mean arterial pressure (MAP) of 50 to 60 $\mathrm{mmHg}$. To ensure rapid recognition of changes in intravascular volume, direct monitoring of blood pressure using a blood pressure transducer in a radial arterial line was combined with real-time observation and communication with the surgeon about bleeding in the surgical field.

The standard anesthetic technique involved induction with propofol with or without a short-acting opiate (eg, fentanyl or alfentanil) and maintenance with a gaseous agent (sevoflurane, desflurane, or isoflurane) with or without remifentanil with positive pressure ventilation. Many patients also received a lumbar epidural with $0.5 \%$ bupivacaine, which was loaded before emergence from anesthesia and which was used for postoperative analgesic effect only and so is unlikely to have influenced the

Table 1. Patient and surgical demographics

\begin{tabular}{|c|c|c|c|}
\hline Variable & $\begin{array}{l}\text { HEA } \\
(\mathrm{n}=102[59 \%])\end{array}$ & $\begin{array}{l}\text { Standard } \\
(\mathrm{n}=72[41 \%])\end{array}$ & $\mathrm{p}$ value \\
\hline \multicolumn{4}{|l|}{ Age (years) } \\
\hline Mean \pm SD & $46 \pm 20$ & $50 \pm 19$ & 0.13 \\
\hline \multicolumn{4}{|l|}{ Gender } \\
\hline Male versus female & $\begin{array}{l}54 \text { versus } 48 \\
(53 \% \text { versus } 47 \%)\end{array}$ & $\begin{array}{l}38 \text { versus } 34 \\
(53 \% \text { versus } 47 \%)\end{array}$ & 0.98 \\
\hline \multicolumn{4}{|l|}{ Tumor volume } \\
\hline Mean \pm SD & $985 \pm 1150$ & $791 \pm 871$ & 0.08 \\
\hline Procedure & & & $0.17^{\dagger}$ \\
\hline Hemipelvectomy & $59(57 \%)$ & $47(65 \%)$ & \\
\hline Type I, I/IV & 18 & 17 & $0.14^{*}$ \\
\hline Type I/II, I/II/III & 14 & 4 & \\
\hline Type II, II/III & 20 & 22 & \\
\hline Type III & 7 & 4 & \\
\hline Sacrectomy & $18(18 \%)$ & $6(9 \%)$ & \\
\hline Hindquarter amputation & $25(25 \%)$ & $19(26 \%)$ & \\
\hline Mean MAP $(\mathrm{mmHg})^{*}$ & $53(47-57)$ & $58(52-63)$ & 0.001 \\
\hline \multicolumn{4}{|l|}{ Duration } \\
\hline Procedure (minutes) ${ }^{*}$ & $167(117-228)$ & $159(120-200)$ & 0.55 \\
\hline HDU stay (hours) ${ }^{*}$ & $20(17-27)$ & $20(17-29)$ & 0.96 \\
\hline Admission (days) ${ }^{*}$ & $17(11-27)$ & $21(13-35)$ & 0.14 \\
\hline
\end{tabular}

* Data presented as median (interquartile range); ${ }^{\dagger}$ comparison between hemipelvectomy, sacrectomy, hindquarter amputation; ${ }^{*}$ comparison between hemipelvectomy types; HEA = hypotensive epidural anesthesia; MAP = mean arterial pressure; HDU = high-dependency unit. 
outcomes of interest in this study. No adrenaline infusion was administered to this group and invasive monitoring of blood pressure was not routinely used.

Antifibrinolytics were used in both groups of patients (hypotensive epidural anesthesia $42.1 \% \mathrm{n}=24$ of 57 [aprotin nine, tranexamic acid 15, none 33] versus the standard anesthetic group $32.6 \% \mathrm{n}=17$ of 53 [aprotin five, tranexamic acid 12, none 35], $\mathrm{p}=0.28$ ). Aprotin was withdrawn in 2007 and since then tranexamic acid has been used, given as an infusion at a rate of $500 \mathrm{mg} /$ hour after a loading dose of $1 \mathrm{~g}$ to a maximal dose of $3 \mathrm{~g}$.

Packed red blood cells were transfused intraoperatively and postoperatively to all patients with a hemoglobin level $<8 \mathrm{mg} / \mathrm{dL}$, ongoing blood loss, and/or signs of intravascular depletion (tachycardia and hypotension). Postoperative transfusion was also given to patients with a hemoglobin level $<9 \mathrm{mg} / \mathrm{dL}$ and signs or symptoms of anemia (fatigue, dizziness, shortness of breath, tachypnea, or tachycardia).

Antibiotic prophylaxis with broad-spectrum coverage including anaerobes (eg, cefuroxime + metronidazole, flucloxacillin + gentamicin + metronidazole) was routinely given at induction and continued for at least 24 hours up to 5 days. Deep vein thrombosis prophylaxis consisted of mechanical measures with compression stockings and sequential compression devices. Routine chemoprophylaxis (eg, low-molecular-weight heparin) was not used.

\section{Data Collection}

Data were collected from patient notes, anesthetic charts, and electronic databases (for pathology results and mortality data and blood results from 2000 onward). Data included patient demographics (age, gender, weight, diagnosis); operation details (type, duration); anesthetic details (technique, MAP, blood and fluid use); and postoperative details (duration of high-dependency unit stay, fluids and blood product use, total duration of hospital stay). Both groups were comparable in terms of age, gender, tumor volume, and operation type (Table 1). Morbidity and mortality data were also collected, including postoperative complications, return to a high-dependency unit, transfer to an intensive care unit, and 90-day mortality. Mortality data were confirmed through a national registry.

Mean intraoperative MAP was calculated from MAP readings from an arterial line taken at least every 15 minutes from the time of the first incision to the time of wound closure. Patients receiving hypotensive epidural anesthesia had a lower mean intraoperative MAP than the standard group (53 $\mathrm{mmHg}$ versus $58 \mathrm{mmHg}, \mathrm{p}=0.001$ ).

Blood loss was calculated from a previously published formula (Fig. 1) taking into account pre- and postoperative
Estimated Blood Loss $=\{[($ Initial Hb - Final Hb $) /($ Mean Hb) $] X$ EBV $\}+$ TBV

$$
\begin{array}{ll}
\text { Initial } \mathrm{Hb} & \text { Preoperative Hemoglobin } \\
\text { Final } \mathrm{Hb} & \text { Postoperative Hemoglobin }(72-96 \text { Hours) } \\
\text { Mean } \mathrm{Hb} & \text { (Initial Hb + Final Hb)/2 } \\
\text { EBV } & \text { Estimated Blood Volume }(65 \mathrm{~mL} / \mathrm{kg} \text { for Men, } 60 \mathrm{~mL} / \mathrm{kg} \text { for Women } \\
\text { TBV } & \text { Total Blood Volume Transfused ( } 300 \mathrm{~mL} \text { per Unit of Packed Red Cells) }
\end{array}
$$

Fig. 1 This is the equation used within the study to calculate the perioperative blood loss $(\mathrm{Hb}=$ hemoglobin; $\mathrm{EBV}=$ estimated blood volume; $\mathrm{TBV}=$ total blood volume).

hemoglobin measurements and total volume of packed red cells transfused. The postoperative hemoglobin measurement used in calculating blood loss was taken 4 to 7 days after surgery and at least 24 hours from the last packed red blood cell transfusion. Estimates of intraoperative blood loss by the operating surgeon or anesthetist involved in the case were not used for the purposes of this study.

\section{Data Analysis}

Patients were divided into two groups depending on the anesthetic technique they received: hypotensive epidural anesthesia or a standard anesthetic technique. After normality testing, appropriate statistical analyses were undertaken. Parametric data are presented as mean $\pm \mathrm{SD}$ and compared using unpaired t-tests. Nonparametric data are presented as median (interquartile range) and compared using Mann-Whitney U-tests (continuous data) and chi square tests (categorical data). Significance was taken at the 5\% level. Data were analyzed using the SPSS program (Version 22.0; IBM SPSS, Armonk, NY, USA).

\section{Results}

Patients treated with hypotensive epidural anesthesia experienced less blood loss compared with the standard anesthetic group $(1457 \mathrm{~mL}, \mathrm{SD} 1721,95 \%$ confidence interval [CI], 1114-1801 versus $2421 \mathrm{~mL}$, SD 2297, 95\% CI, 1877-2965; $\mathrm{p}=0.003$; Table 2). Patients receiving hypotensive epidural anesthesia also received on average fewer packed red cell transfusions (2.7 units, SD 2.9, 95\% CI, 2.1-3.2 versus 3.9 units, SD 4.4, 95\% CI, 2.9-5.0; $\mathrm{p}=$ $0.03)$.

There were no differences with the numbers available in the proportions of patients experiencing end-organ injury $(7 \%, \mathrm{n}=$ seven of 102 versus $6 \%, \mathrm{n}=$ four of $72, \mathrm{p}=0.72)$ (Table 2). There was also no difference in duration of operation, duration of high-dependency unit stay, or total admission duration between the two groups (Table 1). However, the number of events was small in both study groups, and the no-difference finding here may well reflect the insufficient sample size. 
Table 2. Blood loss, transfusion requirements, and rate of end-organ injury

\begin{tabular}{llll}
\hline Variable & HEA & Standard & p value \\
\hline Blood loss (mL)* & $1457 \mathrm{~mL}$ & $2421 \mathrm{~mL}$ & 0.003 \\
& $($ SD $1721 ; 95 \%$ CI, 1114-1801) & (SD 2297; 95\% CI, 1877-2965) \\
Red cells transfused (units)* & 2.7 units & 3.9 units & (SD 4.4; 95\% CI, 2.9-5.0) \\
& $($ SD 2.9; 95\% CI, 2.1-3.2) & $\mathrm{n}=4(4 \%)$ & $\mathrm{n}=2(3 \%)$ \\
End-organ injury & $\mathrm{n}=7(7 \%)$ & $\mathrm{n}=0(0 \%)$ & 0.72 \\
Acute kidney injury & $\mathrm{n}=6(6 \%)$ & $\mathrm{n}=2(3 \%)$ & 0.47 \\
Cerebrovascular accidents & $\mathrm{n}=0(0 \%)$ & $\mathrm{n}=1(1 \%)$ & 0.57 \\
Acute coronary syndromes & &
\end{tabular}

* Data presented as mean; HEA = hypotensive epidural anesthesia; $\mathrm{CI}=$ confidence interval.

Table 3. Details of patients who died within 90 days of operation

\begin{tabular}{|c|c|c|c|}
\hline Patient details & Patient 1 & Patient 2 & Patient 3 \\
\hline Age (years) & 73 & 82 & 10 \\
\hline Gender & Male & Male & Male \\
\hline Diagnosis & Dedifferentiated chondrosarcoma & Chondrosarcoma & Ewing's sarcoma \\
\hline Operation & Hemipelvectomy & Hemipelvectomy & Hemipelvectomy \\
\hline Anesthetic group & Standard & HEA & HEA \\
\hline Days postoperative & 13 & 30 & 37 \\
\hline Cause of death & Cardiac arrest secondary to MI & Wound sepsis and pneumonia & Chemotherapy complications* \\
\hline Bloods loss (mL) & 3108 & 2168 & 217 \\
\hline
\end{tabular}

* Cause given on death certificate; HEA = hypotensive epidural anesthesia; MI = myocardial infarction.

With the very few events observed, there were no differences between the study groups in terms of the 90-day mortality rate $(1.9 \%, \mathrm{n}=$ two of 102 in hypotensive epidural anesthesia versus $1.3 \%, \mathrm{n}=$ one of 72 in the standard anesthetic group, 95\%; $\mathrm{p}=0.77$; Table 3 ). The overall 90-day mortality in the series was $1.72 \%$ (three patients of 174).

\section{Discussion}

Surgery for pelvic and sacral tumors is technically demanding and can be associated with substantial blood loss $[13,21]$. The anesthetic challenges are numerous, as detailed by Molnar et al. [13] in a study of 49 patients undergoing hemipelvectomy with massive blood loss and resulting in coagulopathy of primary concern. Hypotensive epidural anesthesia is a technique that uses an extensive epidural block and low-dose epinephrine infusion resulting in arterial hypotension but with preservation of central venous pressure, controlled heart rate, and cardiac output [9]. It has been shown to be effective in reducing blood loss in THA $[10,15,18-20]$. Its use in surgery for pelvic and sacral tumors has not previously been reported. We found that hypotensive epidural anesthesia in bone and soft tissue pelvic tumor surgery resulted in less blood loss and fewer blood units transfused on average per patient. Importantly, 90-day mortality and incidence of end-organ damage did not appear to be increased (albeit the numbers here were small) when compared with a group receiving standard anesthetic techniques. We note that despite the name of the technique, the decreased blood loss is not solely accounted for by the lower blood pressure. Hypotensive epidural anesthesia includes spontaneous breathing, hyperdynamic circulation, and decreased peripheral resistance, causing venous hypotension with the effect of reducing the filling of the pelvic veins so that they are less likely to be damaged at surgery, thus reducing the bleeding. We have demonstrated a reduction in blood loss and transfusion use in patients undergoing pelvic tumor resection with hypotensive epidural anesthesia in keeping with previous studies evaluating its use in other procedures [3, 17, 22].

We acknowledge several limitations to this study. First, the retrospective nature of our study limited our options for measuring blood loss. We chose to calculate blood loss using recorded pre- and postoperative hemoglobin levels 
and amount of red blood cells transfused previously published for use in arthroplasty [4]. Alternative methods used to estimate blood loss in hemipelvectomy include estimation by surgeons [21], weighing surgical swabs, and recording blood present in suction devices. These operations often result in loss of blood onto drapes, gowns, and the operating room floor, making measuring techniques both subjective and inaccurate [13]. For these reasons, we feel that calculating blood loss using the objective and measurable variables mentioned is an acceptable method for this study, which avoids assessor bias from the involved surgeon or anesthesiologist. The need for transfusion is a notoriously difficult parameter to quantify because it is not only dependent on the quantifiable measures detailed, but also patient-reported, subjective outcomes that will vary between patients. This is a limitation of the study that we have tried to address through quantifiable measures of the need for postoperative transfusion but must acknowledge as a flaw in the study.

This study is also limited by the lack of randomization by technique. The anesthetic technique used was primarily determined by the anesthesiologist to which the case was assigned and, although we cannot completely rule out the possibility of one group having "easier" procedures, selection bias was addressed by the groups having similarsized tumors, proportion of hemipelvectomies and sacrectomies performed, and procedure times pointing to comparable distribution of cases in terms of complexity (Table 1). The hypotensive epidural anesthesia technique was carried out by a limited number of specialist pelvic anesthetists $(n=2)$. In contrast, the standard technique was administered by a nonspecialist orthopaedic anesthesiologist $(\mathrm{n}=16)$ who individually did not routinely perform $(<$ 10 cases) pelvic tumor surgery. While working in a tertiary orthopaedic center meant that all anesthetists had experience in working with orthopaedic oncology patients, we cannot exclude the impact of experience in operations around this particularly challenging site as a possible confounding factor with the two anesthetists performing hypotensive epidural anesthesia accounting for the majority of the pelvic surgeries. This highlights the importance of performing such complex surgery in designated specialist centers with specialist surgeons and anesthetists alike who perform high volumes of these procedures.

Hypotensive epidural anesthesia is a technique already established in arthroplasty, where it has been shown to reduce blood loss and allogeneic transfusion use [9, 15]. Hypotensive anesthesia has also been studied in spinal surgery and maxillofacial surgery where it has demonstrated reductions in blood loss and transfusion use when used in conjunction with antifibrinolytics and topical hemostasis [6]. Another study on its use in oral and maxillofacial surgery demonstrated a reduction blood loss, transfusion use, and operative time [2].

Reports of blood loss and transfusion after pelvic tumor resection are limited. In comparison to previous reports, we demonstrated less blood loss in both hypotensive epidural anesthesia and conventional anesthesia groups. Molnar et al. [13] reported a median transfusion use of 7 units in 49 patients undergoing hemipelvectomy. Senchenkov et al. [16] reported transfusion use with an average of 13.4 units during external hemipelvectomy. This compares with a median transfusion of 2 units in the hypotensive epidural anesthesia group and 3 units in the standard anesthesia group in the current study. There may be several reasons for these differences. First, the unit is one of only five designated bone sarcoma centers in the United Kingdom and sees a high volume of pelvic sarcoma cases (approximately 30-40 each year). We have adapted to accommodate this demand with designated specialist pelvic surgeons and anesthetists. This is reflected in a high level of expertise in managing these highly challenging cases. Second, in a proportion of both anesthesia technique groups, a continuous infusion of tranexamic acid was administered, which has been shown to reduce postoperative blood loss [10].

Despite the positive effect on bleeding, concerns surrounding the risks of hypotensive epidural anesthesia remain. Potential risks of prolonged intraoperative hypotension include hypoperfusion and injury of the vital organs. Although we present a limited sample, our study found no difference in the rates of end-organ injury between the two groups. These findings concur with previous studies examining these risks in both maxillofacial and arthroplasty surgery $[2,20]$. Studies also show that hypotensive epidural anesthesia can be safely used in higher risk patients, including the elderly, and patients with hypertension, ischemic heart disease, or chronic renal impairment [7, 17]. Lubrano et al. [11] demonstrated a transient tubular dysfunction after induced hypotension with propofol and remifentanil (without concurrent adrenaline); however, a clear link to permanent renal dysfunction has not been demonstrated [17]. Specific concerns regarding spinal cord ischemia caused by prolonged periods of hypotension with neuraxial anesthetics is also well documented; studies have demonstrated a MAP of at least $60 \mathrm{mmHg}$ is required to safely maintain spinal cord blood flow [14]. It must be stressed that these studies have reviewed standard neuraxial anesthesia with uncontrolled hypotension. In contrast, hypotensive epidural anesthesia creates a temporary complete cardiac sympathectomy, which allows controlled venous hypotension while maintaining cardiac output and tissue perfusion. The control over physiological parameters creates challenges in detecting intraoperative hypovolemia, 
which may be masked by the administration of the adrenaline infusion (maintaining cardiac output such that a tachycardic response is not mounted). The attending anesthetist must actively monitor blood loss during surgery and replace volume as necessary. Similarly, the adrenaline infusion is controlling the pulse rate and sudden withdrawal can lead to precipitous reduction in cardiac output such that the infusion must be weaned gradually as the epidural sympathectomy effect wears off at the end of the procedure. For these reasons, significant morbidity could result if the technique was used by a physician not experienced in its use.

In addition to low morbidity, with the small numbers available, we saw no differences between the groups in terms of 90-day mortality. However, there were three deaths, seemingly from other causes. These patients are complex, often elderly, and the other treatments they receive can also increase the risk that they might experience a serious complication or death. Although we did not see increased permanent end-organ dysfunction or postoperative mortality at 90 days with our small sample population, further studies are recommended to evaluate the safety of this technique in pelvic tumor surgery.

We found that hypotensive epidural anesthesia resulted in less blood loss, fewer transfusions, and no apparent increase in serious complications; however, the anesthesiologists involved were extremely experienced in this technique. From our encouraging results, we recommend that future collaborative studies possibly in the form of a prospective registry of pelvic tumors treated with surgery be undertaken to confirm the safety of this technique.

\section{References}

1. Cata JP, Gottumukkala V. Blood loss and massive transfusion in patients undergoing major oncological surgery: what do we know? ISRN Anesthesiology. 2012;2012:1-11.

2. Choi WS, Samman N. Risks and benefits of deliberate hypotension in anaesthesia: a systematic review. Int J Oral Maxillofac Surg. 2008;37:687-703.

3. Danninger T. The impact of hypotensive epidural anesthesia on distal and proximal tissue perfusion in patients undergoing total hip arthroplasty. J Anesth Clin Res. 2013;4:366.

4. Guay J. Postoperative pain significantly influences postoperative blood loss in patients undergoing total knee replacement. Pain Med. 2006;7:476-482.

5. Guo W, Li D, Tang X, Yang Y, Ji T. Reconstruction with modular hemipelvic prostheses for periacetabular tumor. Clin Orthop Relat Res. 2007;461:180-188.

6. Hassan N, Halanski M, Wincek J, Reischman D, Sanfilippo D, Rajasekaran S, Wells C, Tabert D, Kurt B, Mitchell D, Huntington J, Cassidy J. Blood management in pediatric spinal deformity surgery: review of a 2-year experience. Transfusion. 2011;51:2133-2141.

7. Ho MC, Beathe JC, Sharrock NE. Hypotensive epidural anesthesia in patients with aortic stenosis undergoing total hip replacement. Reg Anesth Pain Med. 2008;33:129-133.
8. Horvath KA, Acker MA, Chang H, Bagiella E, Smith PK, Iribarne A, Kron IL, Lackner P, Argenziano M, Ascheim DD, Gelijns AC, Michler RE, Van Patten D, Puskas JD, O'Sullivan K, Kliniewski D, Jeffries NO, O'Gara PT, Moskowitz AJ, Blackstone EH. Blood transfusion and infection after cardiac surgery. Ann Thorac Surg. 2013;95:2194-2201.

9. Juelsgaard P, Larsen UT, Sørensen JV, Madsen F, Søballe K. Hypotensive epidural anesthesia in total knee replacement without tourniquet: reduced blood loss and transfusion. Reg Anesth Pain Med. 2001;26:105-110.

10. Lee Y-C, Park S-J, Kim J-S, Cho C-H. Effect of tranexamic acid on reducing postoperative blood loss in combined hypotensive epidural anesthesia and general anesthesia for total hip replacement. J Clin Anesth. 2013;25:393-398.

11. Lubrano R, Marandola M, Antonucci A, Tellan G, Elli M, Santulli M, Lauria D, Giuliana G, Delogu G. Hypotensive anesthesia with propofol and remifentanil: protective effect of alpha-tocopherol on renal function. J Clin Anesth. 2008;20:164-169.

12. Möhnle P, Snyder-Ramos SA, Miao Y, Kulier A, Böttiger BW, Levin J, Mangano DT; Multicenter Study of Perioperative Ischemia (McSPI) Research Group. Postoperative red blood cell transfusion and morbid outcome in uncomplicated cardiac surgery patients. Intensive Care Med. 2011;37:97-109.

13. Molnar R, Emery G, Choong PFM. Anaesthesia for hemipelvectomy-a series of 49 cases. Anaesth Intensive Care. 2007;35:536-543.

14. Neal JM, Barrington MJ, Brull R, Hadzic A, Hebl JR, Horlocker TT, Huntoon MA, Kopp SL, Rathmell JP, Watson JC. The Second ASRA Practice Advisory on Neurologic Complications Associated With Regional Anesthesia and Pain Medicine. Executive Summary 2015. Reg Anesth Pain Med. 2015;40:401-430

15. Niemi TT, Pitkänen M, Syrjälä M, Rosenberg PH. Comparison of hypotensive epidural anaesthesia and spinal anaesthesia on blood loss and coagulation during and after total hip arthroplasty. Acta Anaesthesiol Scand. 2000;44:457-464.

16. Senchenkov A, Moran SL, Petty PM, Knoetgen J, Clay RP, Bite U, Barnes SA, Sim FH. Predictors of complications and outcomes of external hemipelvectomy wounds: account of 160 consecutive cases. Ann Surg Oncol. 2008;15:355-363.

17. Sharrock NE, Beksac B, Flynn E, Go G, Della Valle AG. Hypotensive epidural anaesthesia in patients with preoperative renal dysfunction undergoing total hip replacement. Br J Anaesth. 2006;96:207-212.

18. Sharrock NE, Cazan MG, Hargett MJ, Williams-Russo P, Wilson PD. Changes in mortality after total hip and knee arthroplasty over a ten-year period. Anesth Analg. 1995;80:242-248.

19. Sharrock NE, Fischer G, Goss S, Flynn E, Go G, Sculco TP, Salvati EA. The early recovery of cognitive function after totalhip replacement under hypotensive epidural anesthesia. Reg Anesth Pain Med. 2005;30:123-127.

20. Sharrock NE, Salvati EA. Hypotensive epidural anesthesia for total hip arthroplasty: a review. Acta Orthop Scand. 1996;67:91107.

21. Tang X, Guo W, Yang R, Tang S, Ji T. Evaluation of blood loss during limb salvage surgery for pelvic tumours. Int Orthop. 2009;33:751-756.

22. Williams-Russo P, Sharrock NE, Mattis S, Liguori GA, Mancuso C, Peterson MG, Hollenberg J, Ranawat C, Salvati E, Sculco T. Randomized trial of hypotensive epidural anesthesia in older adults. Anesthesiology. 1999;91:926-935.

23. Woolley AL, Haughey BH, Hogikyan ND, Schechtman KB, Gates GA, Goldenberg JL. Effect of blood transfusion on recurrence of head and neck carcinoma: retrospective review and meta-analysis. Ann Otol Rhinol Laryngol. 1992;101:724-730. 